\title{
Hesperetin protects against inflammatory response and cardiac fibrosis in postmyocardial infarction mice by inhibiting nuclear factor $\kappa B$ signaling pathway
}

\author{
BING WANG ${ }^{1}$, LIANGHAI LI ${ }^{2}$, PING JIN ${ }^{2}$, MENGQIU LI $^{2}$ and JIANGUO LI ${ }^{1}$ \\ ${ }^{1}$ Department of Critical Care Medicine, Zhongnan Hospital of Wuhan University, Wuhan, Hubei 430071; \\ ${ }^{2}$ Department of Critical Care Medicine, Jingzhou Center Hospital, Jingzhou, Hubei 434020, P.R. China
}

Received April 26, 2016; Accepted March 17, 2017

DOI: $10.3892 /$ etm.2017.4729

\begin{abstract}
Cardiac inflammation and cardiac fibrosis are important parts of cardiac remodeling following myocardial infarction (MI), which may be the basic mechanisms of the development of chronic heart failure. The nuclear factor (NF) $-\kappa \mathrm{B}$ signaling pathway promotes cardiac inflammation and fibrosis. It has reported that hesperetin inhibits cardiac remodeling induced by pressure overload in mice. However, it has remained elusive whether and how hesperetin has a role in cardiac fibrosis post-MI. Therefore, a mouse model of MI was established by left anterior descending coronary artery ligation. Mice received hesperetin (30 mg/kg/day) or vehicle after surgery. After 8 weeks, all mice underwent echocardiography to evaluate cardiac function. Gene expression of cardiac fibrosis markers such as connective tissue growth factor (CTGF) as well as collagen I and III, and histological analysis were applied to determine the level of cardiac fibrosis. The expression of inflammatory markers such as tumor necrosis factor (TNF)- $\alpha$, interleukin (IL)- $1 \beta$ and IL- 6 were assessed by reverse-transcription quantitative PCR and ELISA, and activation of the NF- $\kappa$ B signaling pathway was detected by western blot analysis. It was found that hesperetin reduced the expression levels of TNF- $\alpha$, IL-1 $\beta$, IL-6 and CTGF as well as collagen I and III. The level of collagen deposition in post-MI myocardium was attenuated with the treatment of hesperetin. In additionally, administration of hesperetin inhibited the activation of the NF- $\mathrm{B}$ signaling pathway. These findings indicated that hesperetin may inhibit cardiac inflammation post-MI through blocking the NF- $\kappa \mathrm{B}$ signaling pathway, which may be a key mechanism via which hesperetin attenuates cardiac fibrosis.
\end{abstract}

Correspondence to: Professor Jianguo Li, Department of Critical Care Medicine, Zhongnan Hospital of Wuhan University, 169 Donghu Road, Wuhan, Hubei 430071, P.R. China

E-mail: drljg1817@163.com

Key words: hesperetin, inflammation, cardiac fibrosis, myocardial infarction

\section{Introduction}

A series of inflammatory responses occur subsequent to myocardial infarction (MI), which is the precondition of myocardial repair and myocardial scarring $(1,2)$. However, excessive and persistent inflammation produces an adverse effect on the myocardium, which leads to myocardial fibrosis of the infarction area and later in the non-infarction area. An early study has found that application of reduced the degree of MI glucocorticoids in dogs due to their anti-inflammatory action, which proves that inflammation has a critical role in MI (3). Subsequent studies have found that macrophages, $\mathrm{T}$ lymphocytes, tumor necrosis factor- $\alpha(\mathrm{TNF}-\alpha)$, interleukin- $1 \beta$ (IL-1 $\beta$ ) and IL-6 all have important roles in the area of MI and the deterioration of cardiac function after MI (4-6). Proinflammatory factors, including TNF- $\alpha$, IL-1 $\beta$ and IL-6, are not continuously expressed in normal myocardial tissue, and the production or up-regulated expression of these factors represents an internal resistance to myocardial injury (1). Ono et al (7) found that 20 weeks after MI, the levels of TNF- $\alpha$, IL- $1 \beta$ and IL- 6 in rat hearts were significantly higher than those in the control group, and that the increased levels of gene expression were positively correlated with the degree of the deterioration of cardiac function and collagen deposition. Furthermore, Matsumoto-Ida et al (8) found that TGF- $\beta 1$ expression was enhanced in rats after MI, which induced cardiomyocyte hypertrophy and collagen deposition and stimulated fibroblasts to produce extracellular matrix and promote myocardial fibrosis. These results suggested that the inflammatory response after MI has an important role in myocardial fibrosis. Therefore, the identification of potential drugs with the ability to inhibit inflammation and myocardial fibrosis to improve cardiac remodeling and function subsequent to MI has an important clinical value.

It has been reported that hesperetin, a flavanone glycoside found in citrus fruit peels, inhibits cardiac remodeling induced by pressure overload in mice (9). Previous studies have demonstrated that hesperetin has significant anti-inflammatory, anti-oxidant, anti-apoptotic and anti-tumor effects (10-12). However, it has remained elusive whether and how hesperetin has a role in the inflammatory response post-MI, particularly following cardiac fibrosis. Therefore, the present study utilized 
a mouse model to induce MI in an attempt to clarify whether hesperetin has protective effects against cardiac fibrosis post-MI via inhibiting the inflammatory response and to identify the possible mechanisms underlying these effects.

\section{Materials and methods}

Materials. Primary antibodies against nuclear factor (NF)- $\kappa \mathrm{B}$ p65 (cat. no. 8242) and phospho-(p)-NF-кB p65 (cat. no. 3039) were obtained from Cell Signaling Technology, Inc. (Danvers, MA, USA). GAPDH antibody (cat. no. MB001) was purchased from Bioworld Technology, Inc. (St. Louis Park, MN, USA). TRIzol was obtained from Invitrogen (Thermo Fisher Scientific, Inc., Waltham, MA, USA). The bicinchoninic acid (BCA) Protein Assay kit was purchased from Pierce (Thermo Fisher Scientific, Inc., Waltham, MA, USA). Hesperetin was obtained from Sigma-Aldrich (Merck KGaA, Darmstadt, Germany). Mouse TNF- $\alpha$ Quantikine ELISA kit (cat. no. MTA00B), Mouse IL-1ß/IL-1F2 Quantikine ELISA kit (cat. no. MLB00C) and Mouse IL-6 Quantikine ELISA kit (cat. no. M6000B) were purchased from R\&D Systems Inc. (Minneapolis, MN, USA).

Left anterior descending coronary artery (LAD) ligation. The present study was approved by the Ethics Committee of Zhongnan Hospital of Wuhan University (Wuhan, China) and performed in accordance with the Guide of Zhongnan Hospital of Wuhan University for the Care and Use of Laboratory Animals. All surgeries were performed in a blinded manner regarding grouping. Adult male C57BL/6 mice (weight, 20-25 g; age, 10-12 weeks; $n=43$ ) were purchased from the Experimental Animal Center of Hubei Province (Wuhan, China) and maintained in the following conditions: Temperature, $24 \pm 2^{\circ} \mathrm{C}$; humidity, $40-70 \%$, exposed to a $12 \mathrm{~h}$ light $/ 12 \mathrm{~h}$ dark cycle and had free access to food and water. Mice were randomized to undergo LAD ligation or sham operation with hesperetin or vehicle treatment. The mice were anesthetized with $1 \%$ pentobarbital sodium $(30 \mathrm{mg} / \mathrm{kg}$; Merck KGaA, Darmstadt, Germany). Thoracotomy was performed via the third left intercostal space. The pericardium was opened and prolene (8-0) was used to ligate the LAD. Visible blanching and hypokinesis of the anterior wall of the left ventricle were indicative of successful ligation. Sham operation consisted of the same procedure without ligation. Animals were divided into four groups: Vehicle-sham $(n=9)$, Hesperetin-sham $(n=10)$, Vehicle-MI (n=12) and Hesperetin-MI $(n=12)$. As described by Deng et al (9), freshly prepared $0.5 \%$ carboxymethylcellulose suspension (vehicle) was administered at a constant volume of $1 \mathrm{ml} / 100 \mathrm{~g}$ body weight by oral gavage once a day. The control group was given the same volume of liquid comprising of only $0.5 \%$ carboxymethylcellulose suspension. The mice received $30 \mathrm{mg} / \mathrm{kg} /$ day of hesperetin or vehicle for 8 weeks.

Assessment of cardiac function. After 8 weeks, cardiac function was evaluated by assessing left ventricular ejection fraction (LVEF) and left ventricular fractional shortening (LVFS) by using an echocardiograph. In addition, the mRNA levels of biomarkers of cardiac function, namely atrial natriuretic peptide (ANP) and brain natriuretic peptide (BNP), were detected.
ELISA. TNF- $\alpha$, IL-1 $\beta$ and IL- 6 levels in the serum of mice were measured using the respective kits according to the manufacturer's instructions. In brief, the samples were added to the wells of the ELISA plates, which were pre-oated with polyclonal antibodies. Subsequent to incubation at room temperature, the plates were washed. TNF- $\alpha$, IL-1 $\beta$ and IL-6 conjugates were then added to each well. The plates were incubated at room temperature and then washed again, and substrate solution was added to each well. The plates were then incubated at room temperature in the dark for color development. After $30 \mathrm{~min}$, stop solution was added to each well and the absorbance was measured at $450 \mathrm{~nm}$ using a microplate reader. The concentrations of TNF- $\alpha$, IL- $1 \beta$ and IL- 6 in each sample were determined by interpolation from the standard curve.

Histological analysis. Picrosirius red (PSR) staining for collagen deposition was performed as described previously (13-16). In brief, hearts were fixed in $10 \%$ formalin after being excised from anesthetized mice. The hearts were then dehydrated and embedded in paraffin using standard histological procedures. Subsequently, these hearts were transversely or longitudinally sectioned at $5-\mu \mathrm{m}$ thickness. The slices were stained with PSR (Hede Biotechnology Co., Ltd., Beijing, China) for histological analysis.

Reverse-transcription quantitative polymerase chain reaction $(R T-q P C R)$. To determine the mRNA levels of ANP, BNP, TNF- $\alpha$, IL-1 $\beta$, IL- 6 and connective tissue growth factor (CTGF) as well as collagen I and III, RT-qPCR analysis was used. In brief, total mRNA was extracted from myocardial tissue using TRIzol, chloroform, isopropanol and 75\% diethyl pyrocarbonate-treated ethanol. Synthesis of complementary (c)DNA was performed by using oligo (dT) primers with the Transcriptor First Strand cDNA Synthesis kit (Roche Diagnostics GmbH, Penzberg, Germany). The composition of the PCR mixture was as follows: $\mathrm{ddH}_{2} \mathrm{O}$, PCR Master Mix (Thermo Fisher Scientific, Inc., Waltham, MA, USA), primers (Sangon Biotech (Shanghai) Co., Ltd., Shanghai, Beijing), reference dye (Roche Diagnostics GmbH, Penzberg, Germany) and (c)DNA. PCR reaction was performed in three stages: $95^{\circ} \mathrm{C}$ for $10 \mathrm{~min}$; a 40 -cycle reaction of $95^{\circ} \mathrm{C}$ for $15 \mathrm{sec}$, and $60^{\circ} \mathrm{C}$ for $30 \mathrm{sec} ; 95^{\circ} \mathrm{C}$ for $15 \mathrm{sec}$ and $60^{\circ} \mathrm{C}$ for $1 \mathrm{~min}$. The expression of selected genes was determined by using SYBR green and normalized against the gene expression of GAPDH, and the $2^{-\Delta \Delta C q}$ method was used for quantification (17). The sequences of primers used in the study were as follows: ANP, forward 5'-ACCTGCTAGACCACCTGGAG-3' and reverse 5'-CCT TGGCTGTTATCTTCGGTACCGG-3'; BNP, forward 5'- GAG GTCACTCCTATCCTCTGG-3' and reverse 5'- GCCATTTCC TCCGACTTTTCTC-3'; TNF- $\alpha$, forward 5'-CATCTTCTC AAAATTCGAGTGACAA-3' and reverse 5'-TGGGAGTAG ACAAGGTACAACCC-3'; IL-1 $\beta$, forward 5'-CCGTGGACC TTCCAGGATGA-3' and reverse 5'-GGGAACGTCACACAC CAGCA-3'; IL-6, forward 5'-ACAACGATGATGCACTT-3' and reverse 5'-CTTGGTCCTTAGCCACT-3'; CTGF, forward 5'-TGACCCCTGCGACCCACA-3' and reverse 5'-TACACC GACCCACCGAAGACACAG-3'; collagen I, forward 5'-AGG CTTCAGTGGTTTGGATG-3' and reverse 5'-CACCAACAG CACCATCGTTA-3'; collagen III, forward 5'-CCCAACCCA 
GAGATCCCATT-3' and reverse 5'-GAAGCACAGGAGCAG GTGTAGA-3'; and GAPDH, forward 5'-ACTCCACTCACG GCAAATTC-3' and reverse 5'-TCTCCATGGTGGTGAAGA CA-3'.

Western blot analysis. Total protein was extracted from the tissues and the protein concentration was quantified using the BCA Protein Assay kit (Pierce; Thermo Fisher Scientific, Inc., Waltham, MA, USA) according to the manufacturer's instructions. Subsequent to separation by $10 \%$ SDS-PAGE, the proteins $(20 \mu \mathrm{g} / \mathrm{lane})$ were subsequently transferred to polyvinylidene fluoride membranes and blocked with 5\% nonfat dry milk at room temperature. Membranes were respectively probed with primary antibodies including NF- $\mathrm{\kappa B}$ p65 (1:1,000; cat. no. 8242; Cell Signaling Technology Inc., Danvers, MA, USA), p-NF-кB p65 (1:1,000; cat. no. 3039; Cell Signaling Technology Inc.) and GAPDH (1:5,000; cat. no. MB001; Bioworld Technology, Inc.) at $4^{\circ} \mathrm{C}$ overnight. Subsequent to washing, membranes were incubated with goat anti rabbit (1:10,000; cat. no. 074-1506; Kirkegaard \& Perry Lab, Inc., Gaithersburg, MD, USA) or goat anti mouse $(1: 10,000$; cat. no. 074-1806; Kirkegaard \& Perry Lab, Inc.) secondary antibodies for $1 \mathrm{~h}$ at room temperature. Following several washes, chemiluminescence of the blots were determined using an enhanced chemiluminescence kit (cat. no. 32209; Thermo Fisher Scientific, Inc., Waltham, MA, USA) and AlphaEaseFC Software 4.0.0 (Alpha Innotech, San Leandro, CA, USA) was employed to analyze the optical density. Monoclonal GAPDH antibody was used as a loading control.

Statistical analysis. Values are expressed as the mean \pm standard deviation. One-way analysis of variance was used for comparisons followed by a least-significant differences test (equal variances assumed) or Tamhane's T2 (equal variances not assumed) using SPSS version 13.0 software (SPSS, Inc., Chicago, IL, USA). P $<0.05$ was considered to indicate a statistically significant difference.

\section{Results}

Hesperetin improves cardiac function in MI mice. A mouse model of MI was established by ligating the left coronary artery. No mortalities occurred in the Vehicle-sham, Hesperetin-sham and Hesperetin-MI mice after surgery for 8 weeks. However, one mouse in the Vehicle-MI group died 2 days after LAD ligation. Cardiac function was assessed by determining LVEF and LVFS via echocadiography. Compared with the Vehicle-MI group, administration of hesperetin improved cardiac function, as shown by an increase of LVEF $(46.43 \pm 2.30$ vs. $30.55 \pm 1.18 \%)$ and LVFS $(30.61 \pm 3.36$ vs. $21.59 \pm 5.35 \%$; Fig. 1). In addition, treatment with hesperetin significantly lowered, the mRNA levels of biomarkers ANP and BNP compared with those in the Vehicle-MI group (Fig. 1). These results demonstrated that hesperetin improved cardiac function in the mice post-MI.

Hesperetin reduces inflammatory responses in MI mice. The inflammatory cytokines TNF- $\alpha$, IL- $1 \beta$ and IL- 6 were increased in mice after MI induced by LAD ligation. However, administration of hesperetin significantly reduced the expression levels of TNF- $\alpha$, IL-1 $1 \beta$ and IL-6 in the serum as well as in the infarcted myocardium compared with those in the Vehicle-MI group (Fig. 2). These results suggested that hesperetin exerted an anti-inflammatory effect in MI mice.

Hesperetin inhibits cardiac fibrosis in MI mice. PSR staining showed that less collagen deposition was present in the Hesperetin-MI group when compared with that in the Vehicle-MI group (Fig. 3A). In addition, cardiac fibrosis was estimated by detecting the mRNA levels of fibrosis markers, including CTGF as well as collagen I and III. While CTGF as well as collagen I and III were increased in the Vehicle-MI group, this was significantly inhibited by administration of hesperetin (Fig. 3B-D). These results demonstrated that hesperetin had a protective effect against cardiac fibrosis in the mice following MI.

Hesperetin prevents activation of the $N F-\kappa B$ signaling pathway in MI mice. In order to clarify the mechanism by which hesperetin inhibits the inflammatory response and cardiac fibrosis in MI mice, the role of the NF-kB signaling pathway in the myocardium was assessed. Western blot analysis showed that the level of NF- $\mathrm{kB}$ p65 phosphorylation was markedly increased in the ischemic myocardium, while NF- $\kappa$ B p 65 phosphorylation was reduced in the Hesperetin-MI group when compared with that in the Vehicle-MI group (Fig. 4). These results suggested that administration of hesperetin exerts anti-inflammatory and anti-fibrotic effects by suppressing NF- $\mathrm{kB}$ activation in MI mice.

\section{Discussion}

While mortality due to cardiovascular diseases has been showing yearly declines, the social burden of cardiovascular diseases remains heavy, with medical costs directly or indirectly caused by cardiovascular and cerebrovascular diseases of up to 500 billion dollars in 2010 (18). MI is the main cause of heart failure and has a high prevalence and mortality and as well as a greater impact on social life and economy than other cardiovascular diseases. After the occurrence of MI, due to ischemic injury, a variety of cytokines are expressed and various signaling pathways are activated, leading to excessive proliferation of cardiac fibroblasts as well as overexpression and disordered deposition of extracellular matrix proteins, which causes inflammation, cardiac fibrosis and heart dysfunction (19). Therefore, inhibition of inflammatory responses may reduce cardiac fibrosis and attenuate the deterioration of MI. The traditional Chinese medicine hesperetin was proven to be useful in treating various inflammatory diseases $(10,20,21)$. The findings of the present study indicated that administration of hesperetin reduces the production of pro-inflammatory cytokines, including TNF- $\alpha$, IL-1 $\beta$ and IL- 6 , in infarcted myocardium and provides protection against cardiac fibrosis by suppressing NF- $\kappa \mathrm{B}$ signaling pathway activation in MI mice.

Hesperetin is a major pharmaceutically active component contained in the peel of citrus fruit and its therapeutic effects are mainly due to its anti-oxidant and free radical-quenching effects; it has anti-allergic, anti-dermatitis and neuroprotective properties, adjusts the function of the cardiovascular system, 



Figure 1. Hesperetin improves cardiac function in MI mice. (A) LVEF and (B) LVFS decreased in the Vehicle-MI group 8 weeks after left anterior descending coronary artery ligation surgery; however, the Hesperetin-MI group displayed better cardiac function. The mRNA levels of (C) ANP and (D) BNP in the hearts of mice following Sham or MI surgery with or without hesperetin treatment. The Vehicle-MI group showed an increase in the mRNA levels of ANP and BNP compared with those in the Vehicle-sham group, which was significantly reduced by hesperetin. Values are expressed as the mean \pm standard deviation. " $\mathrm{P}<0.05$ vs. Vehicle-sham group; $\mathrm{P}<0.05$ vs. Hesperetin-sham group; ${ }^{\circledR} \mathrm{P}<0.05$ vs. Vehicle-MI group. LVEF, left ventricular ejection fraction; LVFS, left ventricular fractional shortening; ANP, atrial natriuretic peptide; BNP, brain natriuretic peptide; MI, myocardial infarction.
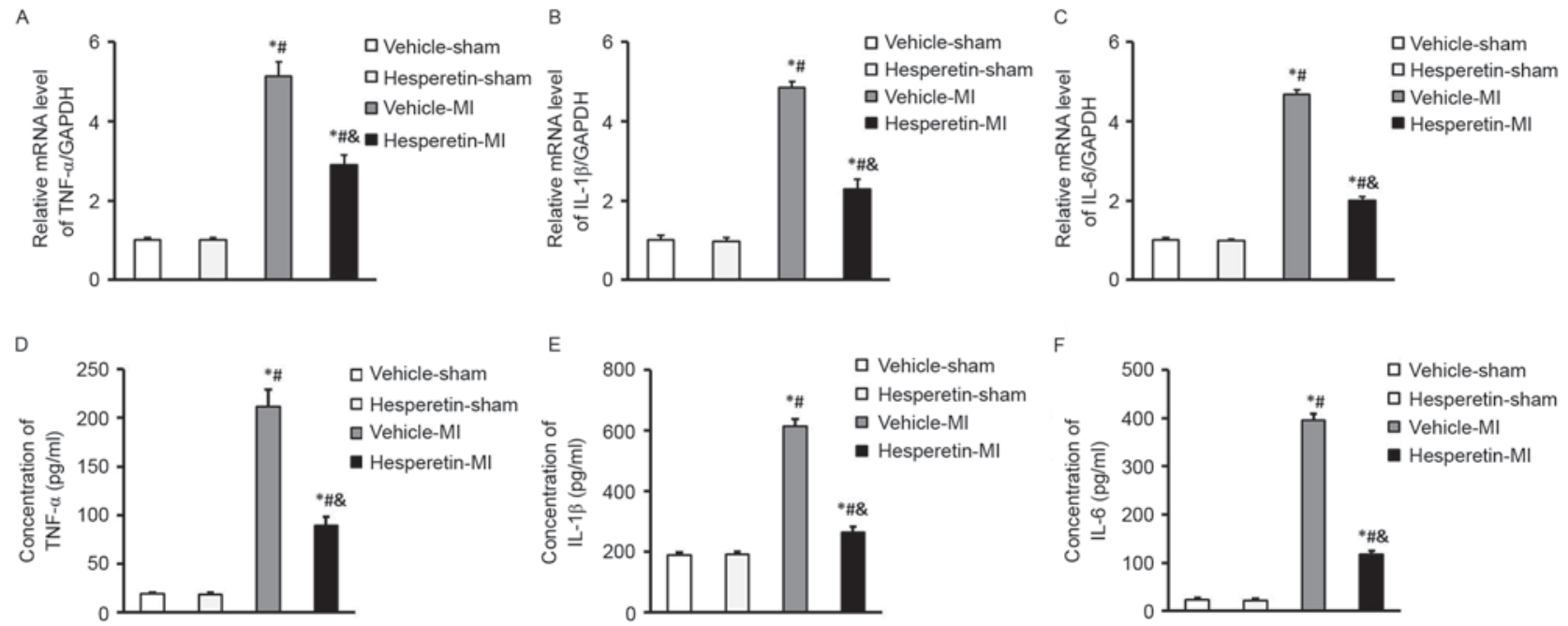

Figure 2. Hesperetin reduces inflammatory responses in MI mice. The mRNA levels of inflammatory cytokines (A) TNF- $\alpha$, (B) IL-1 $\beta$ and (C) IL-6 in the hearts of mice following Sham or MI surgery with or without hesperetin treatment. The levels of inflammatory cytokines (D) TNF- $\alpha$, (E) IL-1 $\beta$ and (F) IL-6 in the serum of the indicated groups determined by ELISA. Compared with those in the Vehicle-sham group, the Vehicle-MI group displayed a higher increase in the levels of TNF- $\alpha$, IL- $1 \beta$ and IL- 6 than the Hesperetin-sham group. Values are expressed as the mean \pm standard deviation. "P $<0.05$ vs. Vehicle-sham group; ${ }^{\#} \mathrm{P}<0.05$ vs. Hesperetin-sham group; ${ }^{\&} \mathrm{P}<0.05$ vs. Vehicle-MI group. MI, myocardial infarction; TNF, tumor necrosis factor; IL, interleukin.

enhances resistance to bacteria and microbes, protects against cancer and genetic toxicity and improves the bioavailability of other drugs $(10,20,21)$. In the cardiovascular system, hesperetin dilates blood vessels, reduces blood lipids, and lowers the permeability of capillaries and brittleness of blood vessels $(11,22)$.
To the best of our knowledge, the present study was the first to show that hesperetin reduced the production of the pro-inflammatory cytokines TNF- $\alpha$, IL- $1 \beta$ and IL- 6 in the infarcted myocardium and serum, and inhibited collagen deposition and the production of the fibrosis biomarkers CTGF as 

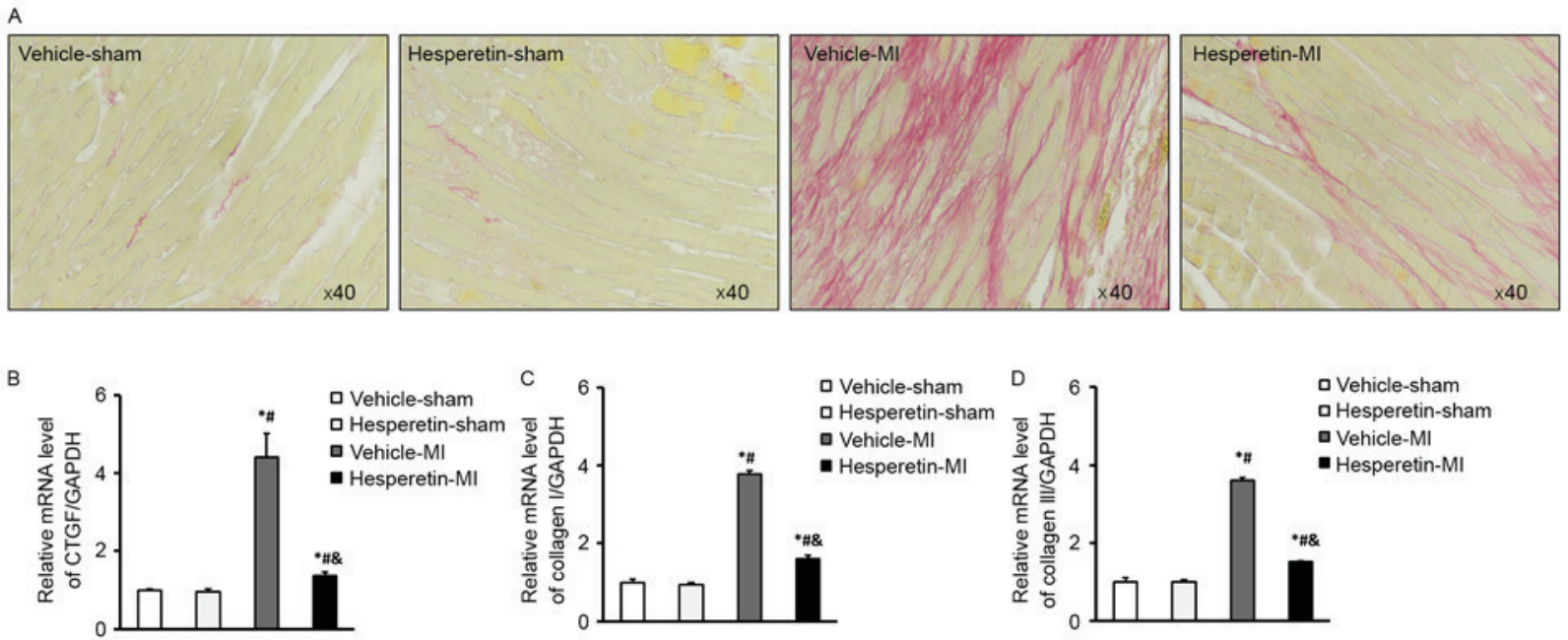

Figure 3. Hesperetin inhibits cardiac fibrosis in MI mice. (A) Increased collagen deposition was present in the Vehicle-MI group at 8 weeks after left anterior descending coronary artery ligation surgery, which was reduced by hesperetin treatment (Picrosirius red staining; magnification, $\mathrm{x} 40$ ). mRNA levels of (B) CTGF, (C) collagen I and (D) collagen III in the hearts of mice following Sham or MI surgery with or without hesperetin treatment. The Vehicle-MI group displayed increased mRNA levels of CTGF as well as collagen I and III when compared to the Vehicle-sham group, which was inhibited by hesperetin treatment. Values are expressed as the mean \pm standard deviation. ${ }^{*} \mathrm{P}<0.05$ vs. Vehicle-sham group; ${ }^{~} \mathrm{P}<0.05$ vs. Hesperetin-sham group; ${ }^{\circledR} \mathrm{P}<0.05$ vs. Vehicle-MI group. MI, myocardial infarction; CTGF, connective tissue growth factor.

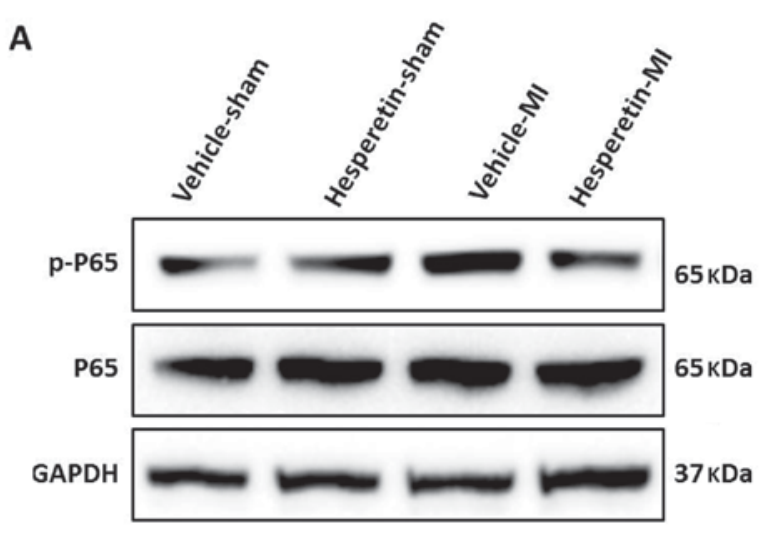

B

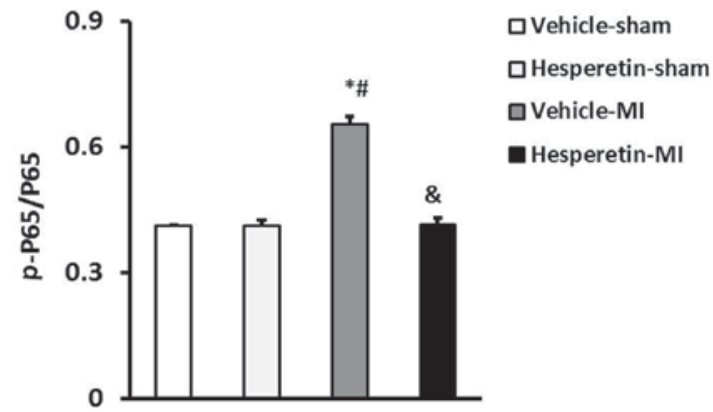

Figure 4. Hesperetin prevents activation of the nuclear factor- $\mathrm{kB}$ signaling pathway in MI mice. (A) Representative western blots of p65 and p-p65 in the hearts of mice with MI. (B) Quantitative results for p-p65 and p65. In the Vehicle-MI group, p-p65 protein was significantly increased compared with that in the Sham groups, which was significantly inhibited by hesperetin treatment. " $\mathrm{P}<0.05$ vs. Vehicle-sham group; ${ }^{\text {" }} \mathrm{P}<0.05$ vs. Hesperetin-sham group; ${ }^{\circledR} \mathrm{P}<0.05$ vs. Vehicle-MI group. MI, myocardial infarction; p-p65, phosphorylated nuclear factor- $\mathrm{\kappa} \mathrm{p} 65$.

well as collagen I and III in the infarcted myocardium. These results demonstrated that hesperetin significantly inhibits the cardiac inflammatory reaction and protects impaired myocardium from exposure to overexpressed inflammatory cytokines, and thus eliminates the effect of inflammatory factors on promoting cardiac fibrosis to then improve cardiac function. Previous studies have confirmed that ischemia of the myocardial tissue increases the levels of pro-inflammatory factors, thus stimulating collagen synthesis and eventually leading to myocardial fibrosis. Inflammatory cytokines and myocardial fibrosis form a complex regulatory network and the levels of inflammatory factors are closely linked to the degree of cardiac fibrosis $(1,2)$. Therefore, the protective effect of hesperetin may be associated with the following mechanisms: First, hesperetin significantly inhibited $\mathrm{NF}-\kappa \mathrm{B}$ signaling pathway activation; $\mathrm{NF}-\kappa \mathrm{B}$ is a key regulator of the inflammatory response, whose activation promotes inflammation and induces the production of pro-inflammatory cytokines, including TNF- $\alpha$, IL-1 $\beta$ and IL-6, and it has been demonstrated that pro-inflammatory cytokines exert their effects on cardiomyocytes through activating $\mathrm{NF}-\kappa \mathrm{B}$ after $\mathrm{MI}(23,24)$. Furthermore, hesperetin inhibited cardiac fibrosis induced by inflammatory factors; inflammatory cytokines not only stimulate the synthesis of extracellular matrix in cardiac fibroblasts, but also reduce the number of cardiomyocytes by causing cardiac necrosis and apoptosis after MI, resulting in cardiac fibrosis (25).

In conclusion, the present study provided evidence for a novel cardioprotective role of hesperetin in a mouse model of MI. This protection was conferred, at least partially, by its anti-inflammatory and anti-fibrotic effects following MI. These findings may provide a basis for a novel therapeutic application of hesperetin in the prevention of cardiac remodeling and improving cardiac function following MI.

\section{Acknowledgements}

This work was supported by a grant from the National Natural Science Foundation of China (grant no. 81571946). 


\section{References}

1. Saxena A, Russo I and Frangogiannis NG: Inflammation as a therapeutic target in myocardial infarction: Learning from past failures to meet future challenges. Transl Res 167: 152-166, 2016.

2. Swirski FK: Inflammation and repair in the ischaemic myocardium. Hamostaseologie 35: 34-36, 2015.

3. Libby P, Maroko PR, Bloor CM, Sobel BE and Braunwald E: Reduction of experimental myocardial infarct size by corticosteroid administration. J Clin Invest 52: 599-607, 1973.

4. Huang M, Yang D, Xiang M and Wang J: Role of interleukin-6 in regulation of immune responses to remodeling after myocardial infarction. Heart Fail Rev 20: 25-38, 2015.

5. Wilkowska A, Pikuła M, Rynkiewicz A, Wdowczyk-Szulc J, Trzonkowski P and Landowski J: Increased plasma pro-inflammatory cytokine concentrations after myocardial infarction and the presence of depression during next 6-months. Psychiatr Pol 49: 455-464, 2015 (In English, Polish).

6. Toldo S, Mezzaroma E, Bressi E, Marchetti C, Carbone S, Sonnino C, Van Tassell BW and Abbate A: Interleukin-1 $\beta$ blockade improves left ventricular systolic/diastolic function and restores contractility reserve in severe ischemic cardiomyopathy in the mouse. J Cardiovasc Pharmacol 64: 1-6, 2014.

7. Ono K, Matsumori A, Shioi T, Furukawa Y and Sasayama S: Cytokine gene expression after myocardial infarction in rat hearts: Possible implication in left ventricular remodeling. Circulation 98: 149-156, 1998.

8. Matsumoto-Ida M, Takimoto Y, Aoyama T, Akao M, Takeda T and Kita T: Activation of TGF-beta1-TAK1-p38 MAPK pathway in spared cardiomyocytes is involved in left ventricular remodeling after myocardial infarction in rats. Am J Physiol Heart Circ Physiol 290: H709-H715, 2006.

9. Deng W, Jiang D, Fang Y, Zhou H, Cheng Z, Lin Y, Zhang R, Zhang J, Pu P, Liu Y, et al: Hesperetin protects against cardiac remodelling induced by pressure overload in mice. J Mol Histol 44: 575-585, 2013.

10. Adan A and Baran Y: The pleiotropic effects of fisetin and hesperetin on human acute promyelocytic leukemia cells are mediated through apoptosis, cell cycle arrest, and alterations in signaling networks. Tumour Biol 36: 8973-8984, 2015.

11. Roohbakhsh A, Parhiz H, Soltani F, Rezaee R and Iranshahi M: Molecular mechanisms behind the biological effects of hesperidin and hesperetin for the prevention of cancer and cardiovascular diseases. Life Sci 124: 64-74, 2015.

12. Sharma M, Akhtar N, Sambhav K, Shete G, Bansal AK and Sharma SS: Emerging potential of citrus flavanones as an antioxidant in diabetes and its complications. Curr Top Med Chem 15: 187-195, 2015.

13. Jiang DS, Li L, Huang L, Gong J, Xia H, Liu X, Wan N, Wei X, Zhu X, Chen Y, et al: Interferon regulatory factor 1 is required for cardiac remodeling in response to pressure overload. Hypertension 64: 77-86, 2014.
14. Jiang DS, Wei X, Zhang XF, Liu Y, Zhang Y, Chen K, Gao L, Zhou H, Zhu XH, Liu PP, et al: IRF8 suppresses pathological cardiac remodelling by inhibiting calcineurin signalling. Nat Commun 5: 3303, 2014

15. Jiang DS, Liu Y, Zhou H, Zhang Y, Zhang XD, Zhang XF, Chen K, Gao L, Peng J, Gong $\mathrm{H}$, et al: Interferon regulatory factor 7 functions as a novel negative regulator of pathological cardiac hypertrophy. Hypertension 63: 713-722, 2014.

16. Jiang DS, Luo YX, Zhang R, Zhang XD, Chen HZ, Zhang Y, Chen K, Zhang SM, Fan GC, Liu PP, et al: Interferon regulatory factor 9 protects against cardiac hypertrophy by targeting myocardin. Hypertension 63: 119-127, 2014.

17. Livak KJ and Schmittgen TD: Analysis of relative gene expression data using real-time quantitative PCR and the 2(-Delta Delta C(T)) Method. Methods 25: 402-408, 2001.

18. WRITING GROUP MEMBERS, Lloyd-Jones D, Adams RJ, Brown TM, Carnethon M, Dai S, De Simone G, Ferguson TB, Ford E, Furie K, et al: Heart disease and stroke statistics-2010 update: A report from the American Heart Association. Circulation 121: e46-e215, 2010.

19. Glezeva N, Horgan S and Baugh JA: Monocyte and macrophage subsets along the continuum to heart failure: Misguided heroes or targetable villains? J Mol Cell Cardiol 89 (Pt B): 136-145, 2015.

20. Giménez-Bastida JA, González-Sarrías A, Vallejo F, Espín JC and Tomás-Barberán FA: Hesperetin and its sulfate and glucuronide metabolites inhibit TNF- $\alpha$ induced human aortic endothelial cell migration and decrease plasminogen activator inhibitor-1 (PAI-1) levels. Food Funct 7: 118-126, 2016.

21. Aswar M, Kute P, Mahajan S, Mahajan U, Nerurkar G and Aswar U: Protective effect of hesperetin in rat model of partial sciatic nerve ligation induced painful neuropathic pain: An evidence of anti-inflammatory and anti-oxidative activity. Pharmacol Biochem Behav 124: 101-107, 2014.

22. Hoek-van den Hil EF, van Schothorst EM, van der Stelt I, Swarts HJ, van Vliet M, Amolo T, Vervoort JJ, Venema D, Hollman PC, Rietjens IM and Keijer J: Direct comparison of metabolic health effects of the flavonoids quercetin, hesperetin, epicatechin, apigenin and anthocyanins in high-fat-diet-fed mice. Genes Nutr 10: 469, 2015.

23. Niu J, Jin Z, Kim H and Kolattukudy PE: MCP-1-induced protein attenuates post-infarct cardiac remodeling and dysfunction through mitigating $\mathrm{NF}-\kappa \mathrm{B}$ activation and suppressing inflammation-associated microRNA expression. Basic Res Cardiol 110: 26, 2015.

24. He Q, Zhou W, Xiong C, Tan G and Chen M: Lycopene attenuates inflammation and apoptosis in post-myocardial infarction remodeling by inhibiting the nuclear factor- $\kappa \mathrm{B}$ signaling pathway. Mol Med Rep 11: 374-378, 2015.

25. Prabhu SD and Frangogiannis NG: The biological basis for cardiac repair after myocardial infarction: From inflammation to fibrosis. Circ Res 119: 91-112, 2016. 